\title{
Low-Profile Vertically Polarized Wearable Antenna with Omni-Directional Radiation
}

\author{
Chunxu Mao, Mohsen Khalily, Vikrant Singh, and Pei Xiao \\ Institute for Communication Systems (ICS), Home of the 5G Innovation Centre (5GIC) \\ University of Surrey, Guildford, GU2 7XH, UK \\ \{c.mao, m.khalily, vikrant.singh, p.xiao\}@surrey.ac.uk
}

\begin{abstract}
In this paper, a single-layer planar antenna with vertical polarization and omni-directional radiation is proposed for wearable applications. The antenna consists of two identical shorted patches which are face-to-face located and fed by a microstrip line at the center. Due to the structural symmetry, the current distribution and electric-field distribution are symmetrical regarding the feed, which result in vertical linear polarization normal to the antenna and omni-directional radiation pattern in the azimuthal plane. To verify the design concept, an antenna prototype operating at $2.45 \mathrm{GHz}$ is designed, fabricated and tested. Measured results concur well with the simulations, showing that the antenna has a good impedance matching, omnidirectional radiation pattern, and vertical polarization in the band of interest. The proposed antenna can be a good candidate for wearable and other wireless communication systems.
\end{abstract}

Keywords - Antenna, vertical polarization, omni-directional, wearable antenna.

\section{INTRODUCTION}

Due to a broad potential application in medical, firefighting, and military, body-centric communication has attracted widely research interest in the past few years. In such a body-centric system, wearable antenna plays an important role to realize the information interchange between the nodes and terminals. During the past decades, many wearable antennas have been reported for off-body communication [1]-[3]. However, the research on on-body wearable antenna is relatively scarce [4][5]. Compared with off-body wearable antennas, there are several technical challenges to be tackled for the on-body wearable antennas. First of all, the on-body antenna is required to have an omni-directional radiation pattern to support the communication between the antenna and the surrounding sensors. Moreover, the antenna is expected to have a vertical polarization, which can better excite the surface wave and creeping wave. Besides, the wearable antennas with improved bandwidth, low profile and low specific absorption rate (SAR) are highly desired.

In this paper, a novel wearable antenna with vertical polarization and omni-directional radiation pattern is proposed for on-body wearable application. The antenna has a single layer structure thus is easy for fabrication. The antenna

This work was supported by UK EPSRC project "MILLIBAN" under Grant EP/P008402/2.

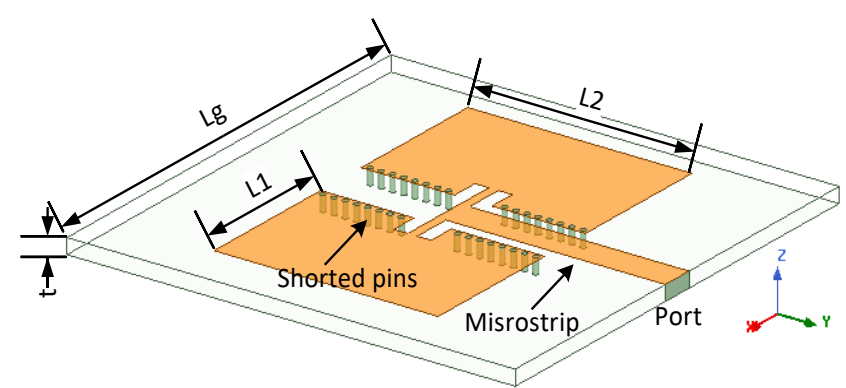

Fig. 1. Configuration of the proposed antenna. $L g=48 \mathrm{~mm}, L 1=16 \mathrm{~mm}$, $L 2=24 \mathrm{~mm}, t=1.525 \mathrm{~mm}$

also adopts an edge-fed method via a microstrip line, which makes it suitable for integration into a garment. To validate the design concept, an antenna prototype is implemented on a rigid substrate for demonstrating the concept based on printed circuit board (PCB) technology. This antenna will be further developed on a flexible material in our future research. The PCB version of this antenna is designed, prototyped, and tested. Measured results agree with the experimental predictions, indicating that the antenna is a promising candidate for on-body wearable applications.

\section{ANTENNA DESIGN}

\section{A. Antenna Configuration}

Fig. 1 shows the geometry of the proposed vertically polarized omni-directional wearable antenna, which is composed of two shorted patches arranged face-to-face. The antenna has a single layer structure with the ground plane printed on the bottom layer of the substrate and the patches printed on the top layer. The patches are shorted to the ground plane. The two patches are connected and fed by a microstrip line in the middle. The inserted metallic vias is purposely used to change the electric field distribution to generate omnidirectional radiation pattern with the vertical polarization. RO4003C substrate with a relative permittivity of 3.55 and thickness of $1.525 \mathrm{~mm}$ is employed, and the optimized parameters are listed in the caption of Fig. 1.

\section{B. Operating Principles}

Fig. 2 shows the simulated current distribution, electric-field distribution, and 3D radiation pattern of the proposed antenna 


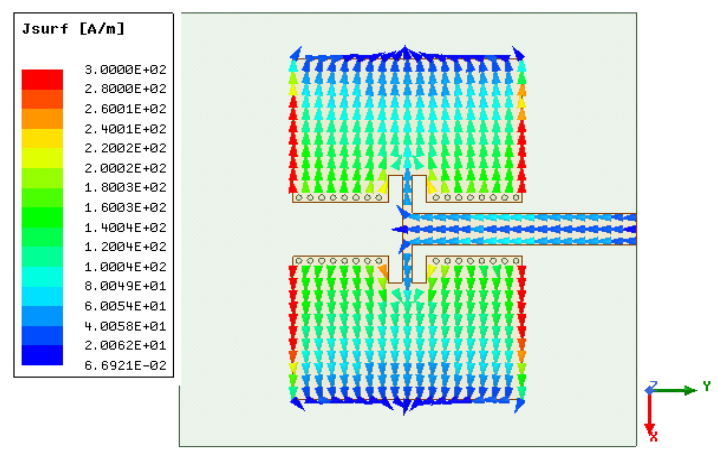

(a)

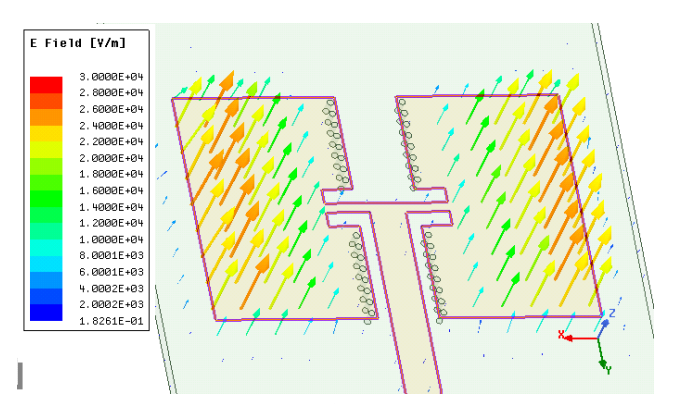

(b)
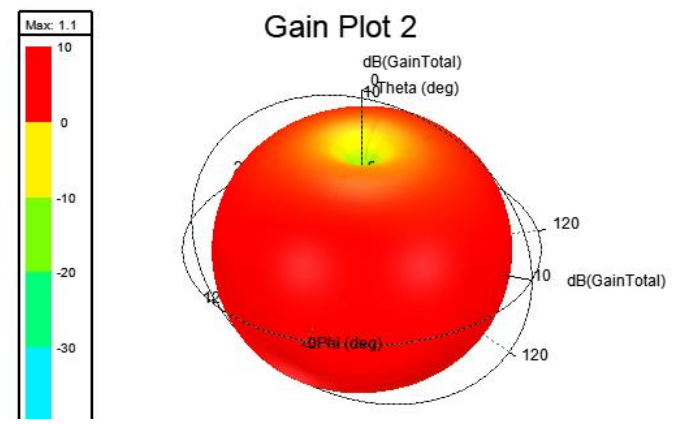

Fig. 2. Simulated results of the proposed antenna at $2.45 \mathrm{GHz}$, (a) current distribution, (b) electric field distribution, (c) 3D radiation pattern.

in the band of interest, $2.45 \mathrm{GHz}$. As observed, the current on the patches are symmetrical along the feeding line due to the introduction of the shorted pins. This unique configuration also results in a good symmetry of the electric field, as shown in Fig. 2(b). The intensity of the electric field is gradually reduced from the edges to the center, such an electric field can generate vertical polarization and omni-directional radiation pattern in the far-field. Fig. 2(c) presents the simulated 3D radiation pattern of the antenna. As can be seen, the antenna exhibits an omni-directional pattern in the azimuth plane with a minimum radiation in the normal direction. The simulated gain is about $1.1 \mathrm{dBi}$.

\section{EXPERIMENTAL RESULTS}

Fig. 3 shows the simulated S-parameters of the proposed wearable antenna. The measured $S_{11}$ agrees reasonably well with the simulation but exhibiting a frequency shift of $15 \mathrm{MHz}$ towards the higher band. The bandwidth is about $20 \mathrm{MHz}$, which can be further improved by increasing the thickness of the substrate. Fig. 4 shows the simulated and measured normalized

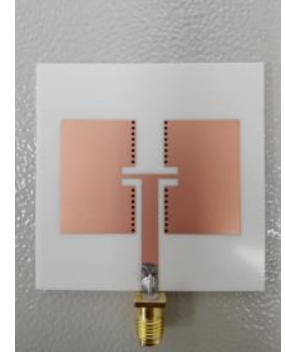

(a)

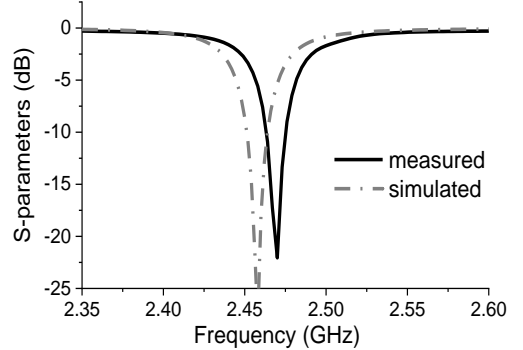

(b)
Fig. 3. (a) Antenna prototype, (b) Simulated and measured S-parameters.

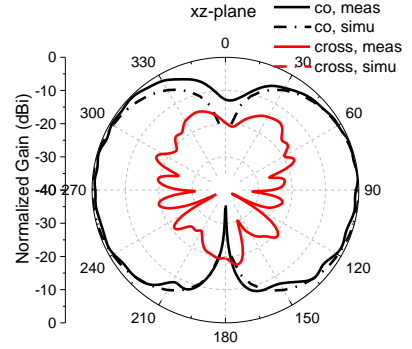

(a)

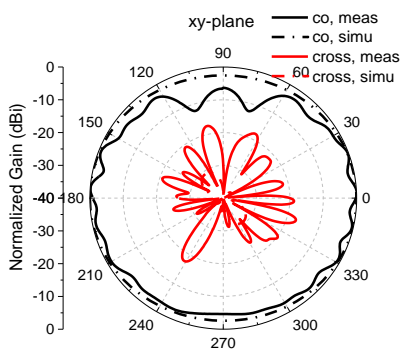

(b)
Fig. 4. Simulated and measured normalized radiation patterns: (a) E-plane, (b) H-plane.

radiation patterns of the antenna in E-, and $\mathrm{H}$-plane, respectively. Results show that the antenna has a good omni-directional radiation performance with a vertical polarization normal to the antenna. It is noted that the radiation pattern is somehow notched due the influence of the SMA connector and cable used in the measurement.

\section{CONCLUSION}

In this paper, a low-profile omni-directional antenna with vertical linear polarization is presented. The omni-directional radiation and vertical polarization are achieved by exciting two face-to-face arranged shorted patches. In this way, the overall electric-field distribution can be tailored. In addition, the antenna is excited via a microstrip, which makes it suitable for wearable system integration. The antenna was implemented through a traditional PCB technology as a proof-of-concept. The proposed antenna was verified experimentally.

\section{REFERENCES}

[1] Z. H. Jiang, Z. Cui, T. Yue, Y. Zhu, and D. H. Werner, "Compact, highly efficient, and gully flexible circularly polarized antenna enabled by silver nanowires for wireless body area networks," IEEE Trans. Biomed. Circuits Syst., vol. 11, no. 4, pp. 920-932, Aug. 2017.

[2] Y. Hong, J. Tak, and J. Choi, "An all-textile SIW cavity-backed circular ring-slot antenna for WBAN applications," IEEE Antennas Wireless Propag. Lett., vol. 15, pp. 1995-1998, 2016.

[3] C. Mao, D. Vital, D. H. Werner, Y. Wu, S. Bhardwaj, "Dual-Polarized Embroidered Textile Armband Antenna Array with Omni-Directional Radiation for On-/Off-Body Wearable Applications," IEEE Trans. Antennas Propag., vol. 67, no. 12, Dec. 2019.

[4] J. Tak, Y. Hong, and J. Choi, "Textile antenna with EBG structure for body surface wave enhancement," Electron. Lett., vol. 51, pp. 1131-1132, 2015.

[5] A. Paraskevopoulos, D. S. Fonseca, R. D. Seager, W. G. Whittow, J. C. Vardaxoglou, and A. A. Alexandridis, "Higher-mode textile patch antenna with embroidered vias for on-body communication," IET Microw. Antennas Propag., vol. 10, no. 7, pp. 802-807, Jul. 2016. 